\title{
Development and Validation of RP-HPLC Method for the Simultaneous Estimation of Hydrochlorothiazide and Losartan Potassium in Tablet Dosage Form
}

\author{
Md. Arif Hossen ${ }^{1}$, Md. Ahsanul Haque ${ }^{1}$, Irin Dewan ${ }^{1}$, A. N. M. Hamidul Kabir ${ }^{2}$, \\ Md. Khalid Hossain ${ }^{3}$ and S. M. Ashraful Islam ${ }^{1}$ \\ ${ }^{1}$ Department of Pharmacy, University of Asia Pacific, Dhanmondi, Dhaka-1209, Bangladesh \\ ${ }^{2}$ Department of Applied Chemistry and Chemical Engineering, Faculty of Engineering, \\ University of Dhaka, Dhaka-1000, Bangladesh \\ ${ }^{3}$ Department of Pharmaceutical Chemistry, Faculty of Pharmacy, University of Dhaka, \\ Dhaka-1000, Bangladesh
}

\begin{abstract}
In the present study, a simple, sensitive and specific liquid chromatography (RP-HPLC) method has been developed and validated for the quantification of hydrochlorothiazide and losartan potassium in tablet dosage form. A shim-pack CLC-ODS column (250 mm X $4.6 \mathrm{~mm}, 5 \mu$ and a mobile phase constituting $0.025 \mathrm{M}$ phosphoric acid solution: acetonitrile (60:40 v/v, pH 3.0 adjusted with $80 \%$ phosphoric acid) were used. The flow rate was 1.5 $\mathrm{ml} / \mathrm{min}$ and detection was carried by using ultraviolet (UV) detector at a wavelength of $254 \mathrm{~nm}$. The retention times of hydrochlorothiazide and losartan potassium were $3.748 \mathrm{~min}$ and $8.790 \mathrm{~min}$, respectively. The peaks of hydrochlorothiazide and losartan potassium were well separated (resolution 22.17). The calibration curves were linear over the concentration range of $80 \%$ to $120 \%$ ( $\mathrm{R}^{2}>0.999$ for both the drugs). The proposed method is accurate with $100.165 \%$ recovery for hydrochlorothiazide and $100.422 \%$ recovery for losartan potassium and precise (\% RSD < 0.5). The proposed method was successfully applied for the estimation of hydrochlorothiazide and losartan potassium in market products (three brands) and potency was found within limit. Therefore, this method can be a convenient and efficient option for the analysis of hydrochlorothiazide and losartan potassium in tablet dosage form.
\end{abstract}

Key words: Hydrochlorothiazide, losartan potassium, method validation, HPLC, quantitative analysis

\section{INTRODUCTION}

Hydrochlorothiazide (HCT), chemically, is 6-chloro3,4-dihydro-2H-1,2,4-benzothiadiazine-7-sulphonamide 1,1-dioxide (Figure 1), is a popular diuretic drug of the thiazide class. It is often used in the treatment of hypertension, congestive heart failure, symptomatic edema and in the prevention of kidney stones.

Correspondence to: S. M. Ashraful Islam

Tel: 880-2-8629367, Extn. 136

Fax: 880-2-9664950

E-mail:ashraf@uap-bd.edu

Dhaka Univ. J. Pharm. Sci. 10(1): 35-42, 2011 (June)<smiles>NS(=O)(=O)c1cc2c(cc1Cl)NCNS2(=O)=O</smiles>

Figure 1. Hydrochlorothiazide

Losartan (LOP), chemically, is (2-butyl-4chloro-1- $\quad\{[2 '-(1 H$-tetrazol-5-yl) biphenyl-4-yl $]$ methyl -1Himidazol-5-yl) methanol. It is an angiotensin II receptor antagonist used mainly to treat hypertension (Figure 2). 


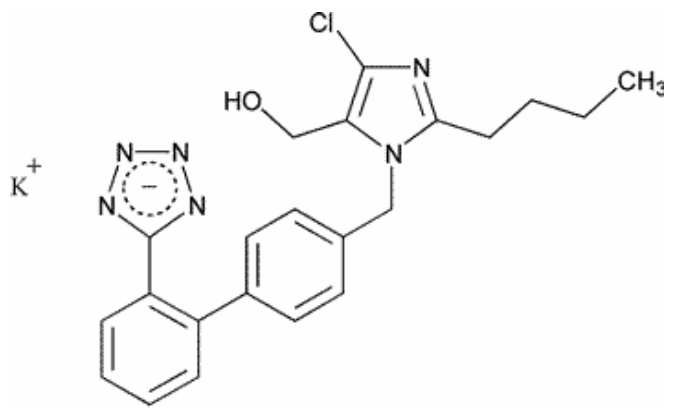

Figure 2. Losartan Potassium

Combination of HCT and LOP is widely prescribed by the physicians due to simple dosing regimens, improved hypertension control, fewer dose-dependent side effects and low cost treatment of hypertension. ${ }^{1}$ So it is essential to develop a simple method for simultaneous estimation of HCT and LOP in combined formulation.

HCT is official in BP and IP, whereas LOP is official in IP and USP ${ }^{2-4}$ The British Pharmacopoeia describes titration method for HCT in bulk and UV method for the assay of hydrochloride tablet. United States Pharmacopeia describes HPLC methods for the determination of LOP both in bulk and tablet. Literature survey revealed that a number of spectroscopic, HPLC methods are reported for the estimation of HCT and LOP individually or in combination with other drugs. ${ }^{5-27}$ Analysis of combination dosage form by spectroscopic method is not free from limitations as various excipients present in the formulation may affect the assay result by providing some absorbance. Very few UV method and HPLC methods are reported for simultaneous estimation of HCT and LOP in tablet dosage form. ${ }^{28-30}$ All these methods are not free from limitations. Therefore, there is still a need to develop a simple HPLC method for the estimation of HCT and LOP in combination dosage form.

The purpose of the present study was to develop and validate an economic, rapid reversed-phase high performance liquid chromatographic method for the quality control of HCT and LOP in pharmaceutical preparations with lower solvent consumption along with the short analytical run time that leads to an environmentally friendly chromatographic procedure and will allow the analysis of a large number of samples in a short period of time. The proposed method can be used for routine analysis of HCT and LOP in combine dosage forms in the pharmaceutical industry.

\section{MATERIALS AND METHODS}

Materials. Hydrochlorothiazide and losartan potassium were provided by Drug International Ltd. Dhaka, Bangladesh. HPLC grade acetonitrile was purchased from E. Merck, Darmstadt, Germany. Phosphoric acid and other reagents were of analytical-reagent grade and purchased from E. Merck, Darmstadt, Germany. Water was deionised and double distilled. Three commercial brands of tablets containing $12.5 \mathrm{mg}$ hydrochlorothiazide and $50 \mathrm{mg}$ losartan potassium were purchased from local drug shops in Dhaka city after checking their manufacturing license numbers, batch numbers, production and expiry dates.

Instrumentation. A Shimadzu (Japan) HPLC system consisting of a CMB-20 Alite system controller, two LC-20AT pumps, SIL-20A autosampler and CTO-10ASVP column oven were used. Ultraviolet detection was achieved at $254 \mathrm{~nm}$ with a SPD-20A UV-VIS detector (Shimadzu, Japan). The drug analyses data were acquired and processed using LC solution (Version 1.3, Shimadzu, Japan) software running under Windows XP on a Pentium PC.

Chromatographic conditions. The mobile phase, a mixture of buffer and acetonitrile (60:40 v/v) pumped at a flow rate of $1.5 \mathrm{ml} / \mathrm{min}$ through the column (C18; $250 \mathrm{~mm} \times 4.6 \mathrm{~mm}, 5 \mu$ shim-pack) at $30^{\circ} \mathrm{C}$. The mobile phase was filtered through a $0.2 \mu$ nylon membrane filter and degassed prior to use under vacuum. Elutions were analyzed by UV detector at a sensitivity of 0.0001 .

Preparation of standard solution. For 100\% standard solution of target concentration $8.0 \mathrm{mg}$ hydrochlorothiazide and $25.0 \mathrm{mg}$ losartan potassium were weighed, dissolved in mobile phase and sonicated. The solution was diluted to produce target concentration for analysis. 80\%, 90\%, 110\%, and $120 \%$ standard solutions of target concentration were 
also prepared in the same way. All the solutions were filtered through $0.2 \mu$ syringe filter.

Preparation of sample solution. 20 tablets were accurately weighed and the average weight was calculated. The tablets were ground to a fine powder with the help of mortar and pestle. Then, $6.25 \mathrm{mg}$ hydrochlorothiazide and $25 \mathrm{mg}$ losartan potassium powder were transferred to a volumetric flask, dissolved in mobile phase and then filtered through filter paper. The filtered solution was further diluted in the mobile phase to make the final concentration of working sample equivalent to $100 \%$ of target concentration.

Validation of HPLC method. Present study was conducted to obtain a new, affordable, cost-effective and convenient method for HPLC determination of HCT and LOP in tablet dosage form. The experiment was carried out according to the official specifications of USP, ICH- 1996, Global Quality Guidelines-2002., 31,32 The method was validated for the parameters like system suitability, selectivity, linearity, accuracy, precision and robustness.

The system suitability was assessed by six replicate analyses of HCT and LOP at a 100\% level to verify the resolution and reproducibility of the chromatographic system. This method was evaluated by analyzing the repeatability of retention time, tailing factor, theoretical plates (Tangent) of the column and resolution between the peaks of HCT and LOP.

Selectivity is the ability to measure accurately and specifically the analyte in the presence of components that may be expected to be present in the sample matrix. $8.0 \mathrm{mg}$ HCT and $25.0 \mathrm{mg}$ LOP were mixed with different amount of placebo formulations (60 mg to $90 \mathrm{mg}$ ), dissolved in mobile phase and diluted to produce $100 \%$ of nominal concentration. The solutions were run and chromatograms were analyzed for retention time, peak area and peak shape to determine selectivity of the method.

The linearity of an analytical method is its ability to elicit that test results are proportional to the concentration of analyte in samples within a given range. This was determined by means of calibration graph using increasing amounts of a standard solutions $(80 \%, 90 \%, 100 \%, 110 \%$, and $120 \%)$ of both HCT and LOP. These standards were tested six times in agreement to the International Conference on Harmonization (ICH). ${ }^{31}$ Calibration curves were constructed and the proposed method was evaluated by its correlation coefficient and intercept value, calculated in the corresponding statistical study (ANOVA) $(p<0.05)$. Characteristic parameters for regression equation $(y=a+b x)$ of the HPLC method were obtained by least squares treatment of the results and these parameters were used to confirm the good linearity of the method developed.

Accuracy indicates the deviation between the mean value found and the true value. Accuracy was determined by means of recovery experiments, by the addition of active drugs to placebo formulations. The accuracy was calculated from the test results as the percentage of the analyte recovered by the assay.

The precision of the method was investigated with respect to repeatability (intra-day), intermediate precision (inter-day variation) and reproducibility (by means of an inter laboratory trial). Repeatability was determined by performing four repeated analysis of the three standard solutions ( $90 \%, 100 \%$ and $110 \%$ of target concentration) of HCT and LOP on the same day, under the same experimental conditions. The intermediate precision of the method was assessed by carrying out the analysis of previous standard solutions on three different days (inter-day) in the same laboratory. For reproducibility the procedure repeated in the Quality Control Laboratory of Drug International Limited. The relative standard deviation (\% RSD) was determined in order to assess the precision of the method.

The robustness of the method was assessed by altering the some experimental conditions such as, by changing the flow rate from 1.4 to $1.6 \mathrm{ml} / \mathrm{min}$, amount of acetonitrile (38\% to $42 \%$,) the temperature of the column $\left(28^{\circ} \mathrm{C}\right.$ to $\left.32^{\circ} \mathrm{C}\right)$ and $\mathrm{pH}(2.9-3.1)$ of the mobile phase. 


\section{RESULTS AND DISCUSSION}

Method development and optimization. The isocratic mode method with UV detection was developed for the determination of the active ingredients, HCT and LOP, at 100\% level. The reversed-phase column, shim-pack CLC, ODS (C18), $250 \mathrm{~mm} \times 4.6 \mathrm{~mm}, 5 \mu$ was tested first. The mobile phase was chosen after several trials with $0.025 \mathrm{M}$ phosphoric acid and acetonitrile in various proportions like 70: 30, 60:40, 50:50, 40: 60, 30:70 and 35:65 at different $\mathrm{pH}$ values. A mobile phase constituting $0.025 \mathrm{M}$ phosphoric acid solution: acetonitrile (60:40 v/v, pH 3.0 adjusted with 80\% phosphoric acid) was selected to achieve maximum separation and sensitivity.

Different flow rates in between 0.50 to 2.0 $\mathrm{ml} / \mathrm{min}$ were studied. A flow rate of $1.5 \mathrm{ml} / \mathrm{min}$ gave an optimal signal to noise ratio with a reasonable separation time.

Wavelength for UV detection was determined by scanning individual and combined standard of HCT and LOP in the UV region. Then HPLC analysis of individual and combined standard was measured at 228, 240, 254, 314, and $332 \mathrm{~nm}$. Finally, all the analysis were done at $254 \mathrm{~nm}$ as at this wavelength, both drugs absorb light better and two peaks could be distinguished properly.

For peak identification a blank sample was injected three times to observe the peak of the blank. No peak was observed. Then samples containing HCT and LOP individually and collectively were injected. Peak area and retention time were found 600820 and $3.470 \mathrm{~min}$ for HCT at $100 \%$ concentration at the width of 50, threshold of 500 and minimum area of 100000 . Whereas peak area of 1408234 with retention time of 8.79 min was observed for single injection of LOP at $100 \%$ nominal concentration at the same width, threshold and minimum area. Solution containing of HCT and LOP with $100 \%$ concentration produced two peaks separately (peak area 601731 for HCT and 1408981 for LOP) with the same retention times $3.47 \mathrm{~min}$ (for HCT) and 8.79 min (for LOP) as found in individual sample run.

\section{Method validation}

System suitability. The system suitability tests were carried out to evaluate the resolution and reproducibility of the system for the analysis. Table 1 represents system suitability results of this method. The system is found suitable in respect of retention time (3.748 min with \% RSD 0.048 for HCT and 8.790 min with \%RSD 0.169 for LOP) mean theoretical plate count (8178 for HCT and 14786 for LOP) and resolution between HCT and LOP (22.16 $\min$ ).

Selectivity. Selectivity is the ability to assess the analyte in the presence of components that may be expected to be present. Typically these might include impurities, degraded products, matrix, etc. Standard solution (100\%) containing both the drugs was injected first. Then drugs solution containing different amount of placebo formulations (60 mg-90 mg) were injected one after another. Figure 3 showed that there was no changes in the retention time of HCT and LOP in the presence of excipients. On the other hand no other peaks other than drugs were found within 12 min run time of the chromatogram which proves the selectivity of the method.

Table 1 Results of system suitability study

\begin{tabular}{|c|c|c|c|c|c|c|c|c|c|c|}
\hline \multirow{2}{*}{$\begin{array}{l}\text { Injection - } \\
\text { no. }\end{array}$} & \multicolumn{4}{|c|}{ HCT } & \multicolumn{6}{|c|}{ LOP } \\
\hline & $\begin{array}{l}\text { Retention } \\
\text { time }\end{array}$ & Area & $\begin{array}{c}\text { Theoretical } \\
\text { plates }\end{array}$ & $\begin{array}{l}\text { Tailing } \\
\text { factor }\end{array}$ & $\begin{array}{l}\text { Retention } \\
\text { time }\end{array}$ & Area & $\begin{array}{c}\text { Theoretical } \\
\text { plates }\end{array}$ & $\begin{array}{l}\text { Tailing } \\
\text { factor }\end{array}$ & Resolution & $\begin{array}{c}\text { Capacity } \\
\text { factor }\end{array}$ \\
\hline 1 & 3.746 & 600820 & 8195 & 1.241 & 8.772 & 1408234 & 14702 & 1.066 & 22.095 & 1.342 \\
\hline 2 & 3.750 & 601731 & 8176 & 1.244 & 8.805 & 1408045 & 14790 & 1.066 & 22.197 & 1.348 \\
\hline 3 & 3.750 & 601335 & 8176 & 1.244 & 8.805 & 1408825 & 14790 & 1.066 & 22.197 & 1.348 \\
\hline 4 & 3.746 & 600820 & 8195 & 1.241 & 8.772 & 1408234 & 14702 & 1.066 & 22.095 & 1.342 \\
\hline 5 & 3.748 & 601654 & 8166 & 1.242 & 8.792 & 1407250 & 14868 & 1.066 & 22.207 & 1.346 \\
\hline 6 & 3.748 & 601608 & 8166 & 1.242 & 8.792 & 1407703 & 14866 & 1.066 & 22.206 & 1.346 \\
\hline Average & 3.748 & 601328 & 8179 & 1.242 & 8.790 & 1408049 & 14786 & 1.066 & 22.166 & 1.345 \\
\hline $\mathrm{SD}$ & 0.002 & 415.515 & 13.21 & 0.001 & 0.015 & 534.557 & 73.871 & 0.000 & 0.055 & 0.003 \\
\hline \%RSD & 0.048 & 0.069 & 0.161 & 0.110 & 0.169 & 0.038 & 0.500 & 0.000 & 0.249 & 0.203 \\
\hline
\end{tabular}




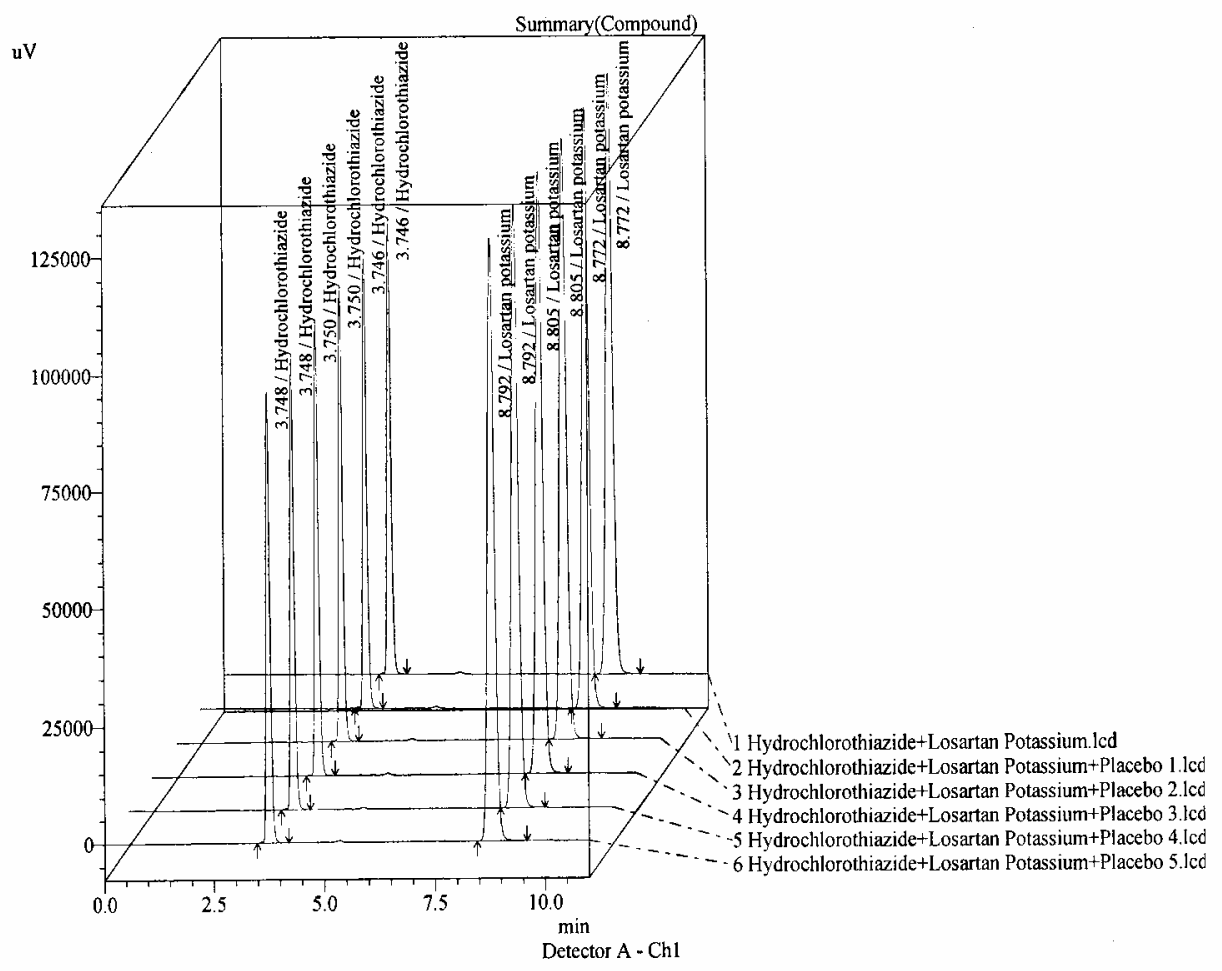

Figure 3. Chromatogram of HCT and LOP along with placebo

Linearity. Linearity of the method was evaluated from the correlation coefficient of calibration curves that were constructed from average peak area of HCT and LOP at different concentration levels (80, 90, 100, 110 and 120\%). Correlation coefficient was 0.999 both for HCT and LOP (Table 2) which prove that the method is linear for HCT and LOP. It means that the response is directly proportional to the concentration of analytes.

Accuracy. Accuracy is generally assessed by analyzing a sample with known concentration and comparing the measured value with the true value. The measured value was obtained by recovery test. Spiked amount of both HCT and LOP were plotted against the recovery amount. In case of HCT \% recovery was $100.165 \pm .078$ (\% RSD 0.078 ) and in case of LOP \% recovery was $100.422 \pm 0.154$ (\% RSD 0.153). All the results indicate that the method is highly accurate.

Repeatability. The measurements for repeatability were done from 9.00 am to $9.00 \mathrm{pm}$. Four determinations of three concentrations across the intended range (90\%, $100 \%$ and $110 \%$ of target concentration) were included in the study. \% RSD of peak areas was calculated for various run. The method is highly precise as \% RSD of peak area was $0.026 \%$ in case of HCT and $0.078 \%$ in case of LOP.

Intermediate precision. The same concentration levels as in the repeatability experiment were used in this study. The results are obtained by 3 concentrations with 4 runs over 3 days. The average peak area obtained at different levels and different days indicate that the method is precise. \% RSD were $0.029 \%$ in case of HCT and $0.21 \%$ in case of LOP.

Reproducibility. Intra day variability was also done in Drug International Limited. The \% RSD of recovery was $0.48 \%$ and $0.44 \%$ for HCT and LOP respectively.

Robustness. 100\% HCT and LOP sample solution was used in this study. The study was performed by making slight variations in flow rate, amount of acetonitrile, temperature and $\mathrm{pH}$ of the 
mobile phase. The results of robustness in the present method are summarized in Table 3 . As the changes are not significant we can say that the method is robust.

Analysis of market products. The proposed method was used to determine the potency of commercially available tablets (three brands) containing $12.5 \mathrm{mg}$ of HCT and $50 \mathrm{mg}$ of LOP. Six replicate determinations $(n=6)$ were carried out and the results are summarized in Table 4.

Table 2. Results of accuracy, precision and linearity study

\begin{tabular}{llcc}
\hline & Validation parameters & HCT & LOP \\
\hline Linearity (regression & & & \\
coefficient-R $\left.{ }^{2}\right)$ & $\mathrm{R}^{2}($ mean $\pm \mathrm{SD})$ & $0.999 \pm 0.0006$ & $0.999 \pm 0.0008$ \\
$\left({ }^{*} \mathrm{Y}=\mathrm{mX}+\mathrm{C}\right)$ & \% RSD ** & 0.060 & 0.080 \\
& Slope (mean $\pm \mathrm{SD})$ & $581476 \pm 6061$ & $1449679 \pm 9992$ \\
Accuracy & \% Recovery & $100.165 \pm .078$ & $100.422 \pm 0.154$ \\
& \% RSD & 0.078 & 0.153 \\
Precision (\% RSD) & Intra day & 0.026 & 0.078 \\
& Inter day & 0.029 & 0.210 \\
& Reproducibility & 0.480 & 0.440 \\
\hline
\end{tabular}

${ }^{*} \mathrm{Y}=\mathrm{mX}+\mathrm{C}$; where $\mathrm{Y}=$ peak area, $\mathrm{m}=$ slope, $\mathrm{X}=$ concentration $(\mu \mathrm{g} / \mathrm{ml})$ and $\mathrm{C}=$ intercept. $* * \% \mathrm{RSD}=$ Relative standard deviation $=$ (Standard deviation X 100)/mean, $\mathrm{R}^{2}=$ Correlation coefficient

Table 3. Results for robustness test of HCT and LOP

\begin{tabular}{lccc}
\hline Parameters & Changes & \% Recovery of HCT & \% Recovery of LOP \\
\hline \multirow{2}{*}{ Flow rate $(\mathrm{ml} / \mathrm{min})$} & 1.4 & 99.8 & 100.25 \\
& 1.5 & 99.9 & 100.14 \\
Column temperature $\left({ }^{0} \mathrm{C}\right)$ & 28 & 99.7 & 99.9 \\
\multirow{2}{*}{ Acetonitrile variation } & 32 & 99.9 & 99.7 \\
& $38 \%$ & 99.5 & 99.7 \\
pH of mobile phase & $42 \%$ & 99.6 & 99.8 \\
& 2.9 & 99.8 & 99.8 \\
\hline
\end{tabular}

Table 4. Analysis of market products by proposed HPLC method

\begin{tabular}{lcccccc}
\hline \multirow{2}{*}{ Assay } & \multicolumn{2}{c}{ Brand-1 } & \multicolumn{2}{c}{ Brand-2 } & \multicolumn{2}{c}{ Brand-3 } \\
\cline { 2 - 7 } & HCT & LOP & HCT & LOP & HCT & LOP \\
\hline 1 & 12.52 & 50.21 & 12.52 & 50.28 & 12.54 & 50.21 \\
2 & 12.34 & 50.13 & 12.23 & 50.43 & 12.43 & 50.32 \\
3 & 12.41 & 50.11 & 12.41 & 50.15 & 12.48 & 50.13 \\
Average & 12.423 & 50.150 & 12.387 & 50.287 & 12.483 & 50.220 \\
\% Potency & 99.387 & 100.300 & 99.093 & 100.573 & 99.867 & 100.440 \\
SD & 0.091 & 0.053 & 0.146 & 0.140 & 0.055 & 0.095 \\
SEM & 0.730 & 0.106 & 1.182 & 0.279 & 0.441 & 0.190 \\
\hline
\end{tabular}

\section{CONCLUSION}

The validation study shows that the developed method is accurate, rapid, precise, reproducible and inexpensive with acceptable correlation co-efficient, RSD (\%) and standard deviations which make it versatile and valuable for simultaneous determination of HCT and LOP in pharmaceutical dosage forms. The proposed method is simple and do not involve laborious time-consuming sample preparation. So this RP-HPLC method can be used for the routine analyses of HCT and LOP in tablets. 


\section{REFERENCES}

1. Prisant, L.M. 2002. Fixed low-dose combination in first line treatment of hypertension. J. Hypertens. 20, S11-S19.

2. British Pharmacopoeia, 2010. Vol. I.. Published by MHRA, UK.

3. Indian Pharmacopoeia. 2007. Vol. II, New Delhi: Govt. of India, Ministry of Health and Family Welfare, Published by the Controller of Publication.

4. United States Pharmacopoeia. 2009. $27^{\text {th }}$ ed. Washington DC: United States Pharmacopoeial Convention Inc.

5. Ulu, S.T. and Saglik, S. 2004. Comparison of UV and second derivative spectrophotometric and HPLC methods for the determination of losartan in tablets. Turk J. Pharm. Sci. 1, 65-75

6. Williams, R.C., Alasandro, M.S., Fasone, V.L., Boucher, R.J. and Edwards, J.F. 1996. Comparison of liquid chromatography, capillary electrophoresis and supercritical fluid chromatography in the determination of losartan potassium drug substance in Cozaar tablets. J. Pharm. Biomed. Anal. 14, 1539-1546.

7. Mccarthy, K. E., Qingxi, W., Tsai, E. W., Gilbert, R. E., Ip D. P. and Brooks M. A. 1998. Determination of losartan and its degradates in COZAAR tablets by reversed phase highperformance thin-layer chromatography. J. Pharm. Biomed. Anal. 17, 671-677.

8. Furtek, C.I. and Lo. M. W. 1992. Simultaneous determination of a novel angiotensin II receptor blocking agent, losartan, and its metabolite in human plasma and urine by highperformance liquid chromatography. J. Chroma. Biomed. Appl. 573, 295-301.

9. Lee, H. Shim, H.O. and Lee, H.S. 1996. Simultaneous determination of losartan and active metabolite EXP3174 in rat plasma by HPLC with column switching. Chromatographia 42, 39-42.

10. Walily, A.F.M., Belal, S.F. and Heaba, E.A. 1997. An improved method for the simultaneous determination of losartan and its major metabolite, EXP3174, in human plasma and urine by high-performance liquid chromatography with fluorescence detection. J. Pharm. Biomed. Anal. 15, 1021-1029.

12. Farthing, D., Sica, D., Fakhry, I., Pedro, A. and Gehr, T.W.B. 1997. Simple high-performance chromatographic method for determination of losartan and E-3174 metabolite in human plasma, urine and dialysate. J. Chromatogr. B. 704, 374-378.

13. Andrea, S., Hildegard S. and Ernst M. 1998. HPLC assays to simultaneously determine the angiotensin-AT1 antagonist losartan as well as its main and active metabolite EXP 3174 in biological material of humans and rats. J. Pharm. Biomed. Anal. 16, 863-873.
14. Prabhakar, A.H. and Giridhar, R. A. 2002. Rapid colorimetric method for the determination of Losartan potassium in bulk and in synthetic mixture for solid dosage form. J. Pharm. Biomed. Anal. 27, 861-6.

15. Erk, N. 2003. Application of first derivative UVspectrophotometry and ratio derivatives pectrophotometry for simultaneous determination of candesartan cilexetil and hydrochlorothiazide. Pharmazie 58, 796-800.

16. Charles, J., Brault, J., Boyer, S., Langlois, C., Cabrero, M.S. and Dubost L. 2003. Simultaneous determination of Irbesartan and hydrochlorothiazide in tablets by derivative spectrophotometry. Anal Lett. 36, 2485-95

17. Nivrutti, M. and Prafulla, B.C. 2009. Simultaneous spectrophotometric estimation of the amlodipine besylate and hydrochlorothiazide. Asian J. Res. Chem. 2, 393-397.

18. Stolarczyk, M., masalanka, A., Krzek, J. and Milczarek, J. 2008. Application of derivative spectrophotometry for determination of enalapril, hydrochlorothiazide and valsartan in complex pharmaceutical preparations. Acta Poloniae Pharm Drug Res. 65, 275-281

19. Satana, E., Altinay, S., Goger, N.G., Ozkan, S.A. and Senturk, Z. 2001. Simultaneous determination of valsartan and hydrochlorothiazide in tablets by first order UV spectrophotometry and liquid chromatography. J. Pharm. Biomed Anal. 25, 1009-1013.

20. Tian, D., Tian, X., Tian, T., Wang, Z. and Mo F. 2008. Simultaneous determination of valsartan and hydrochlorothiazide in tablets by RP - HPLC. Indian $J$. Pharm. Sci. 70, 372-374.

21. Taomin, H., Zhong H., Yang, B., Luping S., Xiaowei Z. and Gengli D. 2006. Simultaneous determination of captopril and hydrochlorothiazide in human plasma by RP - HPLC from linear gradient elution. J. Pharm. Biomed. Anal. 41, 644-648.

22. Sivakumar, T., Venkatesan, P., Manavalan, R and Valliappan, K. 2007. Development of HPLC method for the simultaneous determination of losartan potassium and atenolol in tablets. Indian J. Pharm. Sci. 69, 154-57.

23. Topale, P.R., Gaikwad, N.J. and Tajane, M.R. 2003. Simultaneous UV-spectrophotometric estimation of losarten potassium and amlodipine in tablet. Indian Drugs. 40, 11921.

24. Priyanka, R.P., Sachin U.R., Dhabale, P.N. and Burade, K.B. 2009. RP - HPLC method for simultaneous estimation of losartan potassium and amlodipine besylate in tablet formulation. Int. J. Chem. Tech. Res. 1, 464-469.

25. Wankhede, S.B., Raka, K.C., Wadkar, S.B. and Chitlange, S.S. 2010. Spectrophotometric and HPLC methods for simultaneous estimation of amlodipine besylate, losartan potassium and hydrochlorothiazide in tablets. Indian $J$. Pharm. Sci. 72, 136-140. 
26. Kavitha, J. and Muralidharan, S. 2010. Development and validation of new method for atenolol, hydrochlorothiazide and losartan potassium by RP-HPLC: Its Application to routine quality control analysis. Int. J. Chem. Tech. Res. 2, 880-884

27. Sathe S. R. and Bar, S. B. 2007. Simultaneous analysis of losartan potassium, atenolol, and hydrochlorothiazide in bulk and in tablets by high-performance thin-layer chromatography with uv absorption densitometry. Acta Chromatography 19, 270-278

28. Lande, N.R., Shektar, B.M., Kadam, S.S. and Dhaneshwar, S.R. 2000. Simultaneous spectrophotometric estimation of losartan potassium and hydrochlorothiazide in tablet dasage form. Indian Drugs. 37, 577-81
29. Dinc, E. and Ustundag, O. 2005. Application of multivariate calibration techniques to HPLC data for quantitative analysis of binary mixture of hydrochlorothiazide and losartan in tablet. Chromatographia 61, 237-44

30. Hertzog, D.L., Cafferty, J.F., Fang, X.G., Tyrell, R.J. and Reed, R.A. 2002. Development and validation of stability indicating HPLC method for simultaneous determination of losartan potassium, hydrochlorothiazide and their degradation product. J. Pharm. Biomed Anal. 30, 747-60.

31. International Conference on Harmonization. Draft Guideline on Validation of Analytical Procedures: 1995. Definitions and Terminology, Federal Register. 60, 11260.

32. Global Quality Guideline. Validation of Analytical Procedures. 2002; Number: G-6.9, Version: 1.0 